\title{
Genetic polymorphism and risk of venous thromboembolism among patients with endometrial hyperplasia
}

\author{
Dobrokhotova YE, Lapina IA, Ozolinya LA, Saprykina LV, Grishin II, Gavrilov MV, Yakubova K and Ramazanova A \\ Department of Obstetrics and Gynecology, N.I. Pirogov Russian National Research Medical University, Moscow, the Russian Federation
}

\begin{abstract}
Objective: Endometrial hyperplasia is the result of hormonal changes linking with natural ovarian failure in the late reproductive and perimenopausal period. The most part of these patients need hormone therapy, but this treatment increase the risk of thrombosis (both arterial and venous), especially that some of our patients have hidden genetic determines of the thrombophilia.

Methods: Data were collected for patients with endometrial hyperplasia from September 2008 to September 2016. Incidence of deep vein thrombosis was only one, after 3 months of hormonal treatment. Fishers exact test was used to estimate the association between the presence of hypercoagulation status and genetic determines of the thrombophilia.

Conclusion: A promising direction of modern medicine is the problem of early prevention of thrombotic complications, based on careful study of these coagulation statuses, and in every case of thrombophilia - identifying the causes of these changes, including the identification of genetic defects of coagulation and fibrinolytic systems.
\end{abstract}

Endometrial hyperplasia - is one of the most common proliferative diseases, developing in the reproductive or perimenopausal period, and may be associated with ovarian dysfunction, uterine fibromyoma, endometriosis, metabolic syndrome and other genital and somatic diseases. Among all gynecological diseases, the incidence of endometrial hyperplastic processes ranges from $18 \%$ to $23 \%$ [1-4]. This pathology usually needs hormone therapy. Hormonal treatment may be expected to increase venous thromboembolism risk as a result of thrombophilia as hereditary and acquired character $[5,6]$. We must pay attention to the cases of deserves latent congenital thrombophilia as a result of genetic defects in the blood coagulation and fibrinolytic systems [7].

Hormone therapy of burdened patients increases the risk of thrombosis (venous and arterial) and venous thromboembolism in several times $[8,9]$. The frequency of congenital thrombophilia in patients with thrombosis, according to some authors, ranges from $75 \%$ to $80 \%[10,11]$.

Therefore, the aim of our study was to identify the frequency of genetic polymorphisms of thrombophilia among patients with endometrial hyperplasia and compared with the group of patients with a history of venous thrombosis. This study is required to complete the understanding of the optimal treatment of gynecological patients especially with the high risk of thromboembolic events.

\section{Materials and methods}

In this prospective study, we analyzed the results of a comprehensive clinical and laboratory examination of 207 patients. Women were divided into three groups. Study group comprised 95 patients. The criteria for inclusion in this group were: morphologically confirmed diagnosis of endometrial hyperplasia with obesity (50 patients) and without obesity (45 patients). The comparison group included 51 patients with history of venous thrombosis and post-thrombotic disease. The criteria of the exclusion in the comparison group are the severe decompensation of extragenital disease. The control group consist of 61 health women, finished their reproductive function. Full clinical and laboratory examination, including laboratory tests, that able to provide differential diagnosis of haemostatic disorders (PAI-1 activity, APTT, PTT, platelets count, aggregation of platelets, markers of hemostasis activation, D-dimer).

Genotyping was performed in the genes F5 (mutation of FV Leiden, factor gene encodes V); F2 (G20210A, prothrombin); MTHFR (C677T, 5,10-methylenetetrahydrofolate reductase), SERPINE1 (4G / 5G, plasminogen activator inhibitor-1), THBS4 (G1186C, thrombospondin-4), F7 (G10976A, factor VII), ITGA2 (C807T, glycoprotein GpIa), ITGB3 (T1565C, alloantigen PI (A2), glycoprotein GpIIIa), as well as ACE (I / D, angiotensin converting enzyme) and VKORC1 (1639G > A) [6].

The source of DNA for genotyping were blood samples, which were collected in containers containing EDTA and stored at $-20^{\circ} \mathrm{C}-$ $80^{\circ} \mathrm{C}$ until DNA extraction. DNA was isolated from peripheral blood

Correspondence to: Julia E. Dobrokhotova, Professor, Doctor of Medical Science, $\mathrm{PhD}$, Head of Department of Obstetrics and Gynecology, Medical Faculty, N.I. Pirogov Russian National Research Medical University, Moscow, the Russian Federation, Tel: 8-(903)-722-63-99; E-mail: pr.dobrohotova@mail.ru

Key words: thrombophilia, genetic polymorphism, endometrial hyperplasia, obesity

Received: March 03, 2017; Accepted: March 14, 2017; Published: March 16, 2017 
lymphocytes using reagent kits Diatom DNA Prep 200, based on the use guanidintiotsionatis and Nucleus-sorbent (Isogene Lab. Ltd, Russia). The isolated DNA was stored in a freezer at $-20^{\circ} \mathrm{C}$. Genotyping was performed using the original test systems developed at the Institute of Bioorganic Chemistry. Shemyakin and Ovchinnikov RAS-based PCR in real time with allele-specific probes.

\section{Results}

The average age of women ranged from $44.85 \pm 7.29$ to $57.02 \pm$ 8.27 years, due to the peculiarities of the sample it was different, as in the control group, we included postmenopausal women. We found that in the comparison group of obese patients (mean BMI=27.7 \pm 4.3 ), occurred significantly less frequently than in the study group (mean $\mathrm{BMI}=31.33 \pm 12.01$ ), which was connected with the peculiarities sampling.

Among the patients of the main group $18 \%$ had hypercoagulability and intravascular coagulation activation before treatment, the causes of which had to be clarified in the course of our study. All of them were screened for antiphospholipid syndrome which was not identified.

To eliminate genetic defects of hemostasis, patients were screened for: F5 (FV Leiden, factor V), F2 (G20210A, prothrombin), MTHFR (C677T, MTHFR), SERPINE1 (4G / 5G, PAI-1), THBS4 (G1186C, thrombospondin-4), F7 (G10976A, factor VII), ITGA2 (C807T, GpIa), ITGB3 (T1565C, alloantigen PI (A2), GpIIIa), as well as ACE (I / D, angiotensin converting enzyme) and VKORC1 (1639G > A).

FV Leiden polymorphism was detected in $4.21 \%$ of cases, those women who had more than 40 years of age, were nulliparous, they did not have a history of operations and hormone therapy. Although FV Leiden mutation usually manifested at a younger age, its presence cannot be discounted, given the significant importance of this mutation in the pathogenesis of thrombosis. Hormone therapy can increase risk of thromboembolic events among this group in several times, up to lethal pulmonary thromboembolism. FV Leiden polymorphism had been detected significantly more often in the group with the history of thrombosis, the frequency of this polymorphism was like in general population among patients with endometrial hyperplasia.

Carriage of allele A at polymorphism G20210A prothrombin gene (F2) is also one of the most frequent causes of congenital thrombophilia. The incidence of the pathological A-allele in the general population is $4.1 \%$, while in our study it met in the range of $2.27 \%$ to $4.21 \%$. Our data fully reflect the general population prevalence. Patients of this group ranged from 42 to 45 years old, non-smokers, not previously treated with hormone therapy, that's why they had not the history of thrombosis. Presence of this polymorphism is associated with high risk of thromboembolic events, especially during hormonal treatment or surgery. In the group with the history of thrombosis it was significantly higher $(9.21 \%)$.

The study of gene polymorphism of 5,10-methylenetetrahydrofolate reductase is very actual. Overwhelming clinical studies have identified elevated plasma homocysteine as important risk factors for cardiovascular disease. Our results showed that incidence of homozygous mutant genotype $\mathrm{T} / \mathrm{T}$ was significantly higher in patients with history of venous thrombosis than in the control group (13.72\%), which is in good agreement with literature data [10].

We also calculated the risk ratio of venous thrombosis, depending on the presence of different alleles in the population and in control group. We can conclude that the risk of thrombosis increases in 1.8 times in the population and 2.1 times - among women with endometrial hyperplasia in cases of $\mathrm{T}$ allele carriers.

Plasminogen activator inhibitor gene encodes a protein that plays a critical role in the regulation of fibrinolysis. When the concentration of PAI-1 become higher, the anticoagulant activity of the fibrinolysis system decreases, this process leads to an increased risk of thrombosis.

Figures 1 and 2 shows the results of genotyping of women with endometrial hyperplasia without obesity and patients with thrombosis. Variant genotype $4 \mathrm{G} / 4 \mathrm{G}$ patients with endometrial hyperplasia met in $22,44 \%(n=11)$ observations, and in women with thrombosis - in $47,06 \%$ $(n=24)$ of cases, these rates differed significantly $\left(\chi^{2}=5.28, p=0.0216\right)$. Genotype 5G / 5G patients with endometrial hyperplasia and nonobese detected in $20.0 \%$ of cases $(n=9)$, and in women with thrombosis was absent, that is also the reliability of the results $\left(\chi^{2}=11,26, p=0.0008\right)$. Heterozygous variant genotype $5 \mathrm{G} / 4 \mathrm{G}$ is also responsible for thrombotic complications, as it includes major $4 \mathrm{G}$-allele. This variant met significantly more often in patients with endometrial hyperplasia without impaired fat metabolism - to $55,56 \%(n=25)$ then among women with venous thrombosis - in $52,94 \%(n=27)$ cases. However, we found a significant additive effect $\left(\chi^{2}=11,28, p=0.0008\right)$, then $4 \mathrm{G}$-allele may have harmful effects in the homozygous or heterozygous state in patients with non-obese endometrial hyperplasia.

To determine the risk of thrombosis in patients with endometrial

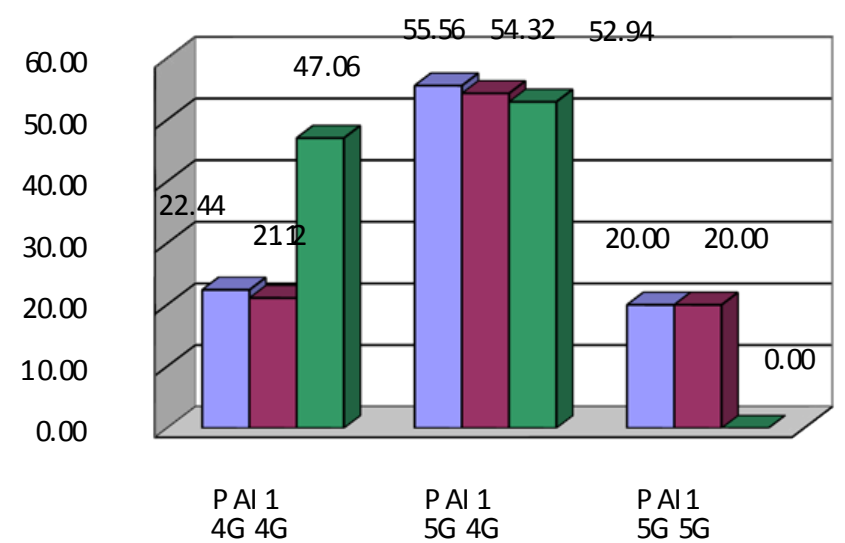

endometrial hyperplasia

nealth women

patients with thrombosis
P Al 1
$4 G$ GG
PAl 1
5 G $4 G$
$5 G 5 G$

Figure 1. Polymorphism in the gene PAI-1 in women with endometrial hyperplasia and no obesity and comparison groups. Note: *The significance of differences is set in comparison with the main group without obesity $\mathrm{p}<0.05$. 


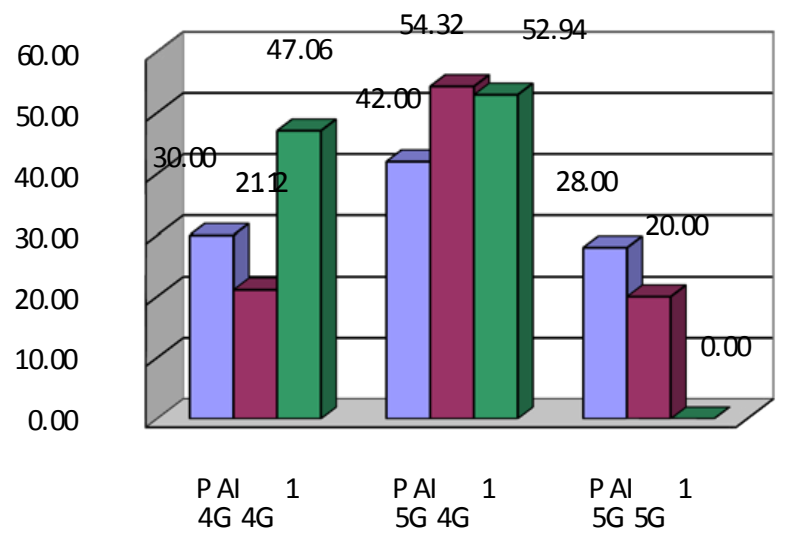

endometrial hyperplasia and obesity

health women

patients with thrombosis

Figure 2. Polymorphism in the gene PAI-1 in women with endometrial hyperplasia and obesity and comparison groups. Note: *The significance of differences is set in comparison with the main group with obesity $\mathrm{p}<0.05$.
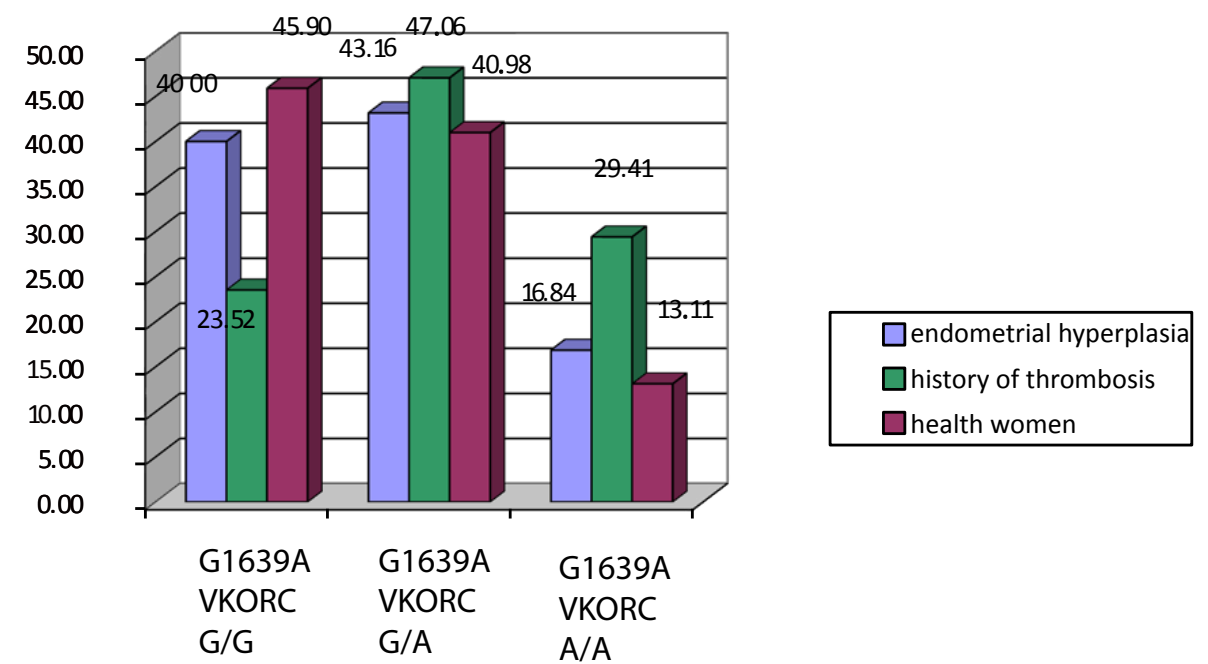

Figure 3. Polymorphism G1639A in the gene VKORC in women with endometrial hyperplasia and comparison groups. Note: *The significance of differences is set in comparison with the main group $\mathrm{p}<0.05$.

hyperplasia, depending on the presence of different alleles of PAI-1 gene, we calculated the risk ratio in carriers of different variants of the gene. On bilateral Fisher's exact test the risk of thrombosis is 1.56 times higher among $4 \mathrm{G}$ allele carriers than in the presence of alternative alleles $5 \mathrm{G}(\mathrm{OR}=1,56, \mathrm{df}=0,9-2,72)$ in women with endometrial hyperplasia. This fact indicates that minor 5G-allele in homozygous state in patients with endometrial hyperplasia is not associated with the development of venous thrombosis.

Therefore, analyzing the results of investigation of PAI-1, we can conclude that there is no significance of differences between health women and patients with endometrial hyperplasia, regardless of the presence of metabolic syndrome.

Heritable defects of Factor VII gene and enzymes involving in the conversion of vitamin $\mathrm{K}$, affect the frequency of the risk of thrombosis and haemorrhage. These embodiments genotypes are more rare in the general population. The results of our study were no exception.

Extremely interesting are the results of investigation of polymorphism G1639A gene VKORC1 (Figure 3). The risk of venous thrombosis in women with allele A is 2.22 times as high as than in the presence of an alternative allele $\mathrm{G}(\mathrm{OR}=2,22, \mathrm{df}=1,25-3,96)$ on bilateral Fisher's exact test. The presence of homozygous genotype A / $A$ increases the risk of venous thrombosis in several times. Incidence of deep vein thrombosis was only one, among this group of patients with genotype A / A of gene VKORC1, after 3 months of hormonal treatment.

Therefore, homozygous genotype A / A of gene VKORC1 is additional risks factors for thrombosis is highly prevalent among patients with endometrial hyperplasia and obesity.

Therefore, we should try to avoid hormone therapy among patient's carrier of homozygous genotype A / A of gene VKORC1 with endometrial hyperplasia, regardless of the presence of metabolic syndrome.

Among all our patients, 17 had identified changes of the haemostatic system only, 10 had severe coagulation changes of hereditary character, 1 case of deep vein thrombosis, 6 - had coagulation of acquired character. 14 of them were from group with endometrial hyperplasia and obesity.

\section{Conclusion}

Thrombophilia is a multicasual disease of hereditary and acquired 
character. We note the importance of identifying thrombophilia before different types of treatment, especially hormonal or surgery.

Patients with high risk of thromboembolic events, requires the investigation of hemostasis, and in the cases of intravascular coagulation (raising PAI-1 activity, APTT, fibrinogen levels and D-dimer) - it is necessary to identify the causes of thrombophilia: genetic polymorphisms, antiphospholipid syndrome, hyperhomocysteinemia, and others.

Therefore, analyzing the results of investigation, although these women may potentially be at increased risk of cardiovascular and thromboembolic events, we cannot rule out the possibility that attenuation of hyperestrogenemia by hormonal treatment may exert some favorable effects on these aspects. But thrombotic risk may differ according to specific hormonal preparations. Therefore, we must pay high attention to the pharmacological approach to endometrial hyperplasia.

However, screening general population for thrombophilia is not justified and so it is not recommended at this time. We hope that our findings stimulate critical discussion among clinicians as to proper means and duration of treatment among patients with endometrial hyperplasia and obesity.

\section{References}

1. Lyon: International Agency for Research on Cancer (2007) Hormonal contraception and postmenopausal hormonal therapy. In: IARC Working Group on the Evaluation of
Carcinogenic Risks in Humans (Ed). IARC Monographs 72: 1-660.

2. Jadoul P, Donnez J (2003) Effects of insulin sensitizing drug metformin on ovarian function, follicular growth and ovulation rate in obese women with oligomenorrhea. Fertil Steril 80: 1315-1322.

3. Darren L Roberts, Caroline Dive, Andrew G Renehan (2010) Biological Mechanisms Linking Obesity and Cancer Risk: New Perspectives. Annual Reviews 61: 301-316.

4. Lai CH, Huang HJ (2006) The role of hormones for the treatment of endometrial hyperplasia and endometrial cancer. Curr Opin Obstet Gynecol 18: 29-34.[Crossref]

5. Grandfather II, Melnichenko GA, Romantsova TI (2004) Pathogenetic aspects of obesity. Obesity and Metabolism 1: 3-9.

6. Ginsburg D (2005) Identifying novel genetic determinants of hemostatic balance. $J$ Thromb Haemost 3: 1561-1568.[Crossref]

7. Guyatt GH, Akl EA, Crowther M, Gutterman DD, Schuünemann HJ, et al. (2012) Antithrombotic Therapy and Prevention of Thrombosis, Executive Summary: 9th ed American College of Chest Physicians Evidence-Based Clinical Practice Guidelines. Chest 141: 7S-47S.

8. Ho WK, Hankey GJ, Quinlan DJ, Eikelboom JW (2006) Risk of recurrent venous thromboembolism in patients with common thrombophilia: a systematic review. Arch Intern Med 166: 729-736. [Crossref]

9. Shapiro S, Dinger J (2010) Risk of venous thromboembolism among users of oral Drospirenone-Containing Oral Contraceptive Pills. J Fam Plann Reprod Health Care36: 33.

10. Patrushev LI (1998) Thrombophilic state and the modern methods of diagnosis. Russian Medical J 6: 3.

11. Diamanti-Kandarakis E, Bergiele A (2001) The influence of obesity on hyperandrogenism and infertility in the female. Obes Rev 2: 231-238.[Crossref]

Copyright: (C2017 Dobrokhotova YE. This is an open-access article distributed under the terms of the Creative Commons Attribution License, which permits unrestricted use, distribution, and reproduction in any medium, provided the original author and source are credited. 